\title{
Design and simulation of a cost-affordable Cosmic Ray Muon Tomographer
}

\author{
J.A. Rengifo ${ }^{a}{ }^{*}$ and J. Bazo $^{a}$ \\ ${ }^{a}$ Sección Física, Departamento de Ciencias, Pontificia Universidad Católica del Perú, \\ Av. Universitaria 1801, Lima 32, Peru \\ E-mail: jrengifo@pucp.edu.pe
}

Atmospheric muons can be used to image a volume due to multiple Coulomb scattering and absorption of different materials. This work presents the design and GEANT4 simulation of a prototype tomographer composed of two detectors, each made of two planes of an array of sensors. The sensors are based on plastic scintillators and silicon photomultipliers targeting new and costaffordable technology. In order to image a volume we study the possibility to discriminate different materials (e.g. lead, concrete, iron, water, aluminum) by measuring the absorption and incoming and outgoing angles of muons passing through these materials. We optimize the geometry and angular resolution of the array using simulations with the aim to scan structures such as large buildings and natural formations with muon tomography.

$37^{\text {th }}$ International Cosmic Ray Conference (ICRC 2021)

July 12th - 23rd, 2021

Online - Berlin, Germany

\footnotetext{
${ }^{*}$ Presenter
} 


\section{Introduction}

Atmospheric muons, because of their high energy, are highly penetrating and non-invasive when they arrive at ground. These muons can provide information, through their energy loss, absorption and scattering, about the density (i.e. composition) of the traversed materials.

In the last 70 years muons have been used to image the inner parts of structures. For instance, the image of a tunnel of the underground railway in London was accomplished by E.P. George in 1956. He used nuclear emulsions as a detector [1]. Later, L.W. Alvarez in 1960 used scintillator counters to image the structure of the second Pyramid of Giza in Cairo, Egypt [2]. Recently, there have been other studies, as the discovery of a void in Khufu's Pyramid in Giza, Egypt [3], which used three types of detectors: photographic films called nuclear emulsions, plastic scintillators and gas detectors. Other projects, as MU-RAY [4], used three planes of plastic scintillators forming a muon telescope to study European volcanoes. In addition, it is possible to look for nuclear material using plastic scintillators with wavelength shifting fiber arrays [5].

In this work we explore muon tomography techniques to identify different materials in an structure with the final aim to image the object. In order to achieve these goals, we design, choosing the best geometrical parameters, and simulate a muon tomographer. The tomographer consists of two detectors each made of two planes. Each plane is designed as an arrange of sensors based on the Desktop Muon Detector (DMD) [6, 7]. The two-plane detectors are arranged before and after the object to be analyzed. The two planes of each detector will allow us to measure the angular deviation with a resolution of $1^{\circ}$.

This tomographer's unit sensor is low cost (i.e. less than 100 USD), easy to build and based on current technology. It is portable considering its small dimensions: its high and width are $0.4 \mathrm{~m}$ and the distance between the two planes is $0.2 \mathrm{~m}$.

\section{Muon Tomography}

Muon Tomography is a non invasive technique to image large inaccessible volumes, such a buildings or natural formations. There are two main methods for applying muon tomography. One method is based on the muon detected counts before and after the object under study (absorption), while the other uses the angular deflection (scattering) after traversing the object to image. These methods are explained next.

\subsection{Absorption method}

Muons are absorbed or attenuated when they pass through matter. The absorption depends on the material and can be used as a method for muon tomography based on the probability of muons passing or not through the object [8].

We can use the ratio between the incoming muons $N_{i}(\phi, \theta)$ and those that have traversed the structure $N_{f}(\phi, \theta)$ measured with the detectors. This ratio depends on the zenith $\theta$ and azimuth $\phi$ angles and change with the type of material. For instance, when muons pass through a block of aluminum the ratio is larger than when the block is made of lead. The correlation between the ratio $\left(N_{f} / N_{i}\right)$ and the material will be tested in our simulation. 


\subsection{Scattering angle method}

When a charged particle interacts with matter, its trajectory is deflected due to Coulomb scattering, which is described by the Rutherford cross section. The distribution of all angles can be approximated by a Gaussian distribution. This scattering angle, $\sigma_{\theta}$, depends on the radiation length, which is a function of the target's charge and mass number [9] as follows:

$$
\sigma_{\theta}=\frac{13.6 \mathrm{MeV}}{\beta c p} \sqrt{\frac{X}{X_{0}}}\left(1+0.038 \ln \left(\frac{X}{X_{0}}\right)\right)
$$

where $p, \beta c$ are the momentum and speed of the incident particle, $X$ is the distance traveled in the material and $X_{0}$ is the radiation length.

\section{Detector design}

We have designed a tomographer which is composed of two detectors, each made of two squared planes of sensor arrays. These arrays consist of $8 \times 8$ sensors based on organic plastic scintillator BC408 made of polystyrene $\left(\left[\mathrm{C}_{6} \mathrm{H}_{5} \mathrm{CHCH}_{2}\right]_{n}\right)$ coupled to a light sensor (e.g. Silicon photomultiplier (SiPM) of $6 \times 6 \mathrm{~mm}^{2}$ ). As an alternative, a more cost affordable light sensor could be based on commercial CMOS webcams. Each plastic cell measures $5 \times 5 \times 1 \mathrm{~cm}^{3}$, thus the total active area is $0.16 \mathrm{~m}^{2}$. Each sensor is isolated inside an aluminum case to reach a better accuracy.

In order to obtain the best resolution and material identification we adjust the geometrical parameters of the measurement setup. These parameters are: the distance between the two planes of each detector $d$ and the distance between the detector and the object under study $D$. We also analyze the width of the object $W$. We will find the best configuration of distances to distinguish between some materials.

\section{GEANT4 simulation}

We used GEANT4 (GEometry ANd Tracking) [10] to simulate the experimental setup in order to understand how our detector will measure muons before and after passing through blocks of different materials. The geometry and materials of the unit sensor were coded in detail and the complete array was accomplished with copies of the unit sensor. In Fig. 1 we show the tomographer's geometry and the experimental setup used to measure the muon counts and deflected angles after passing through a block. As an example, a $4 \mathrm{GeV}$ muon is emitted in a straight line from the center of the detector. When the muon passes through the plastic scintillator, photons are emitted and contained inside the unit sensor. The muon track is deviated inside of the lead block and reconstructed with the set of sensor planes after it.

We simulated for each run 1000 muons at $4 \mathrm{GeV}$, which is the typical muon energy at ground. A more realistic simulation would include energy and angular dependence of the flux, which would be our next step. The muon beam is aimed in a straight line and passes through the detectors and the block. In each run we changed the width and material of the block. The widths of the simulated blocks were 50,100, 150, 200 and $250 \mathrm{~cm}$. For a larger width, muons that pass through lead were mostly absorbed leaving no enough events for a statistically significant result. The simulated materials were air, water $\left(\mathrm{H}_{2} \mathrm{O}\right)$, concrete, aluminium $(\mathrm{Al})$, iron $(\mathrm{Fe})$ and lead $(\mathrm{Pb})$. 


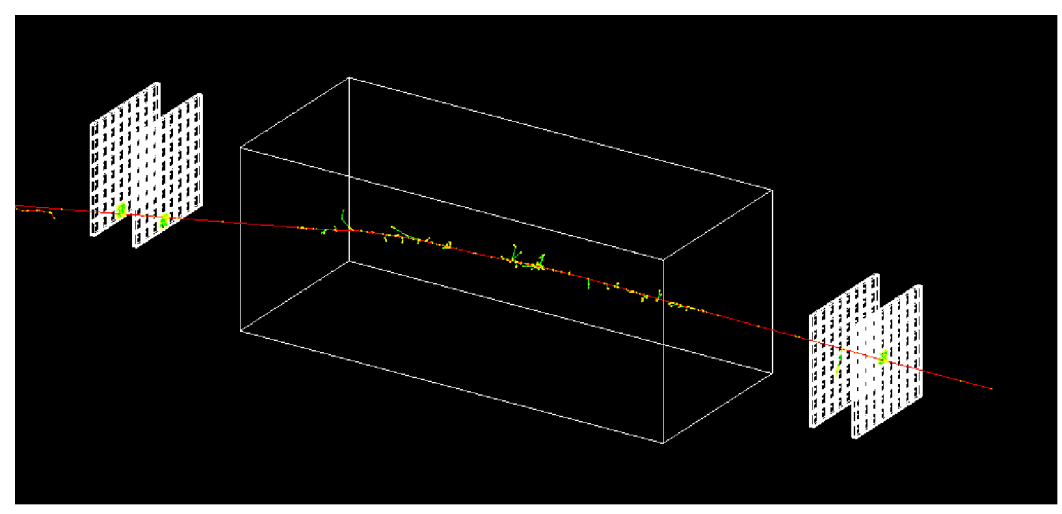

Figure 1: GEANT4 simulation of the muon tomographer made of a set of two sensor planes before and two after the analyzed block. The width of the lead block was $2.5 \mathrm{~m}$. A $4 \mathrm{GeV}$ muon event is simulated. Red lines represent negative charged particles (e.g. the muon track and electrons), while the green lines are photons produced by muon interactions in the plastic scintillator and block, and the yellow points represent step points or physical processes.

We calculated the scattering angle in the following way. We identify the muon hit positions by the emitted scintillation photons in each plane and calculate the corresponding vector that joins both sensors in each detector plane. Using a vector before and another after the block, we calculated the angles of all particles that reached the other side of the block. The muon events were calculated counting the photons detected by the plastic scintillator before and after passing the block.

\section{Results}

First, we used the absorption method to find differences between materials. Using our simulation we count the times that photons are produced by muons that have passed through the detectors. We calculate the ratio between the counts of muons detected before and after passing the studied block. In Fig. 2 we show this ratio as a function of the width of the blocks for the best geometrical parameters (i.e. $d=20 \mathrm{~cm}$ and $D=100 \mathrm{~cm}$ ) for each material. We observe that it is possible to differentiate between lead and iron for certain widths. However, the ratios for aluminum, concrete, water and air are compatible within the statistical error.

In order to quantify the difference between the materials we calculate the number of sigmas between ratios for a given block width. In Fig. 3 we show the number of sigmas as a function of the analyzed object width. We conclude that with this configuration it is possible to differentiate at $5 \sigma$ a block of lead with a width larger than $150 \mathrm{~cm}$ and a block of iron of $250 \mathrm{~cm}$.

Using the scattering angle method we test the same configurations of the detectors and objects. The angular resolution is related to the width of the unit sensor and the distance between the detector and the object $(D)$. In order to achieve a $1^{\circ}$ angular resolution, we calculate that $D$ must be $2.8 \mathrm{~m}$. Thus, to cover the whole scattering angle distribution we need to rotate the second detector. This would be possible using a mechanic structure with a pivot and a stepper motor. The exposure time needed to achieve the simulated number of events depends on the detector surface, the distance 


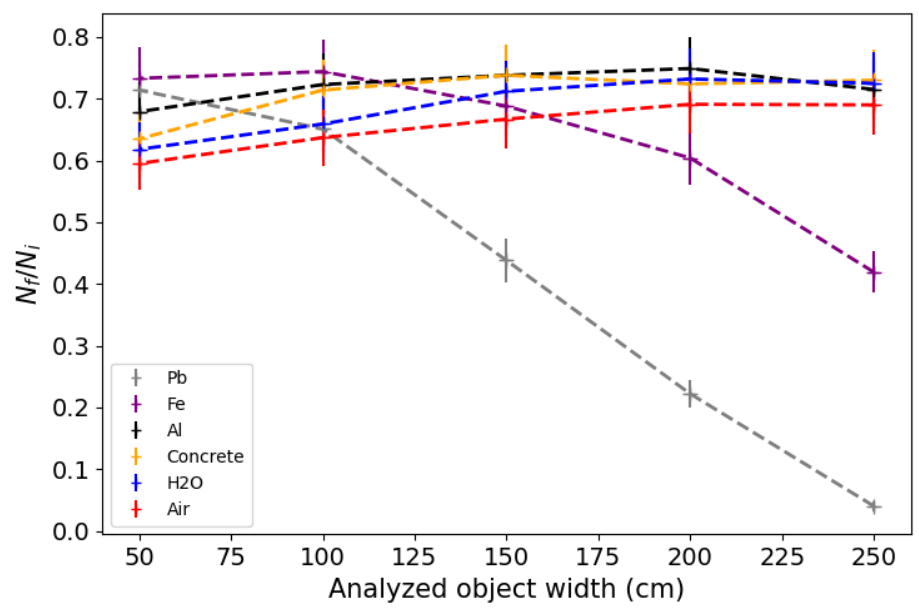

Figure 2: Simulated ratio between incoming muon counts $\left(N_{i}\right)$ and after passing through the block $\left(N_{f}\right)$ versus the width of blocks of different materials: lead $(\mathrm{Pb})$, iron $(\mathrm{Fe})$, aluminum $(\mathrm{Al})$, concrete, water and air
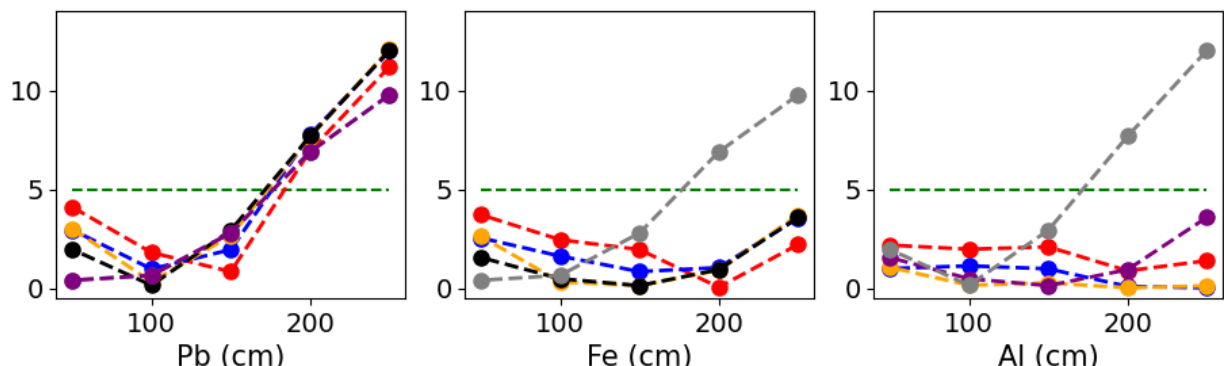

0
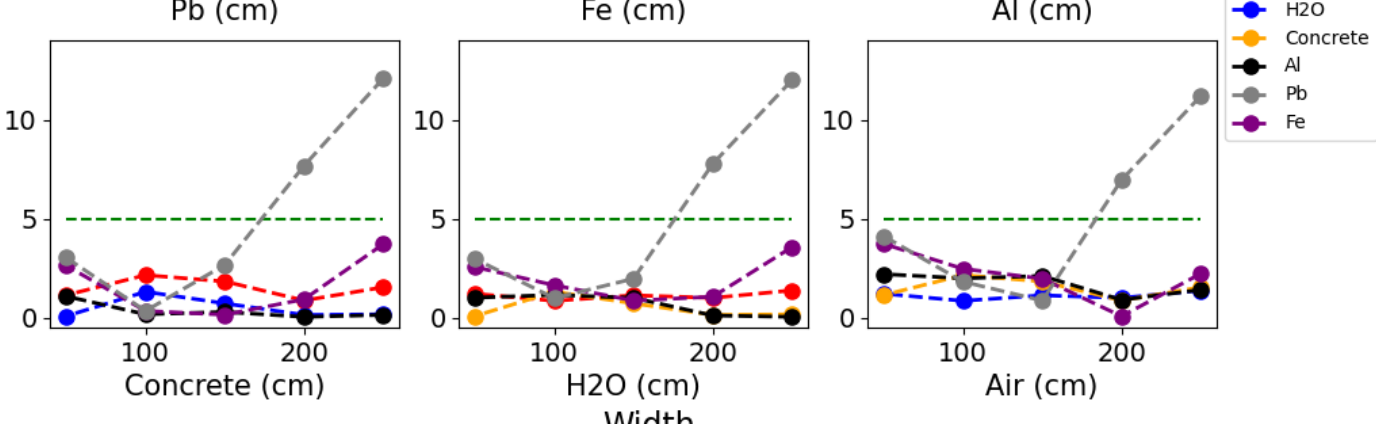

Figure 3: Number of sigmas versus analyzed object width for each material compared with one another. The green line represents $5 \sigma$ 


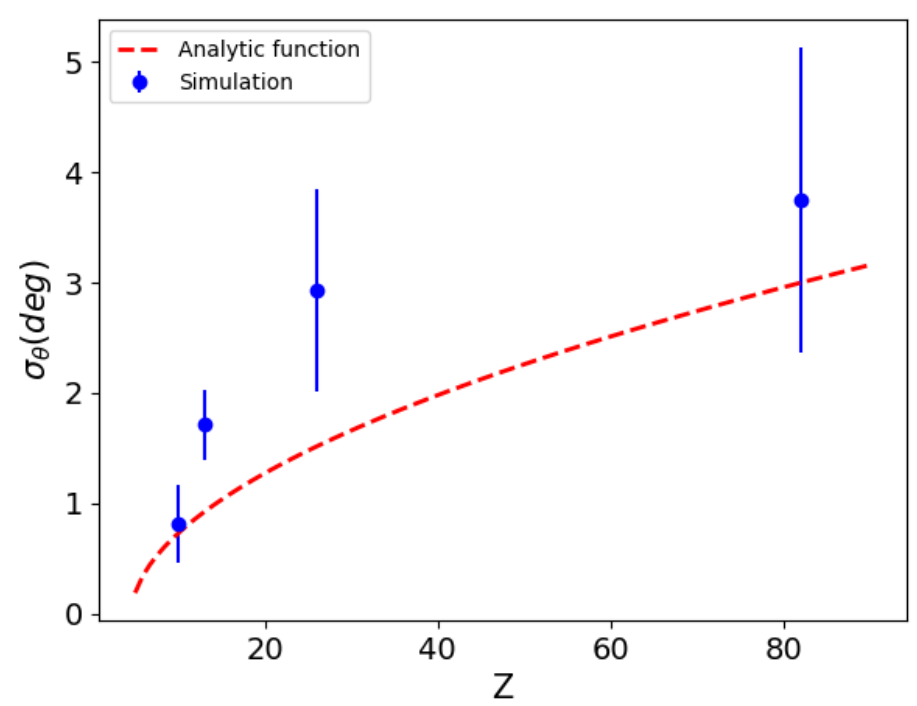

Figure 4: Mean scattering angle as a function of $Z$ for the analytic function (Eq. 1) in red and result from simulation (blue points) based on the $\sigma$ from the Gaussian fit of the scattering angle distribution.

from detector to object and on the azimuthal and zenith angles. In order to obtain 1000 events at 1 ${ }^{\circ}$ of angular resolution the measuring time is approximately 3 hours.

We find as well that it is possible to distinguish blocks of lead and iron from one another. In Fig. 5 we show the scattering angle distribution for most deviated tracks after passing through the different materials using the configuration $d=20 \mathrm{~cm}, D=60 \mathrm{~cm}$ and $W=200 \mathrm{~cm}$. The distribution of the lighter materials are centered close to zero degrees, as expected. For this distance, the angular resolution, represented by the bin size $\left(1^{\circ}\right)$, could not be directly achieved by the density of unit sensors in the array. This can only be done at a larger distance, as explained before.

In Fig. 4 we compare the analytic function (Eq. 1) with the values calculated with a Gaussian fit to the scattering angle distribution from the simulation. The behaviour with $\mathrm{Z}$ is reproduced. However, the mean simulated scattering angle is larger, though compatible within a two sigma deviation from the formula.

To quantify the differences between materials we calculated the p-value using the T-test, which is shown in Fig. 6. We observe that an object made of lead or iron are similar. However, we can differentiate them from water, aluminum, concrete or air. For instance, the p-value result comparing lead and aluminum is 0.005 , but for lead and iron is 0.502 .

\section{Conclusions}

A muon tomographer has been designed and simulated using GEANT4, based on plastic scintillator and SiPM units. An array of $8 \times 8$ sensors conforms a plane. This tomographer has two detector each having two instrumented planes in order to reproduce a track. Each sensor is covered with an aluminum case to isolate the photons from other units and the surroundings. 


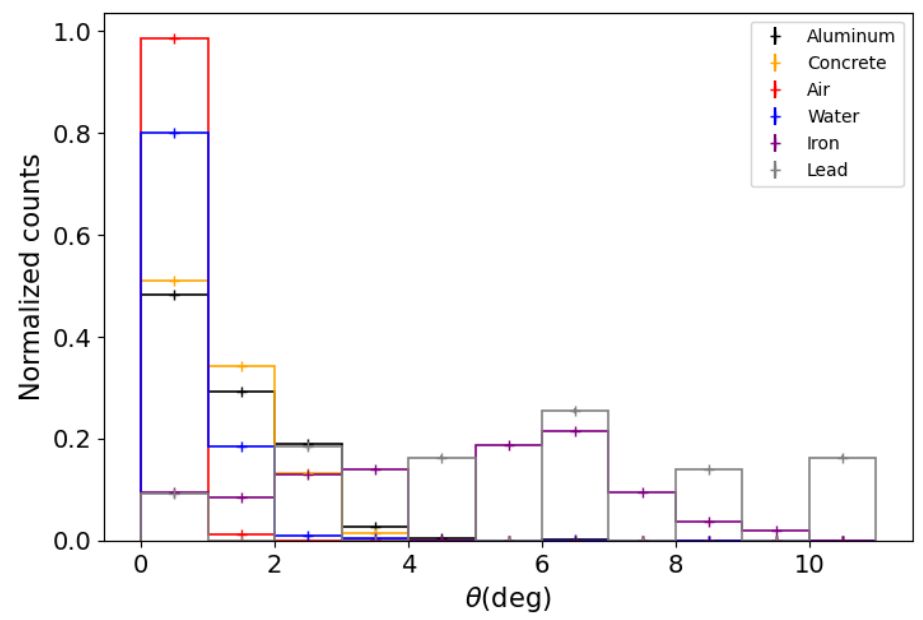

Figure 5: Scattering angle distribution for each material represented with different colors. Configuration $d=20 \mathrm{~cm}, D=60 \mathrm{~cm}$ and $W=200 \mathrm{~cm}$.
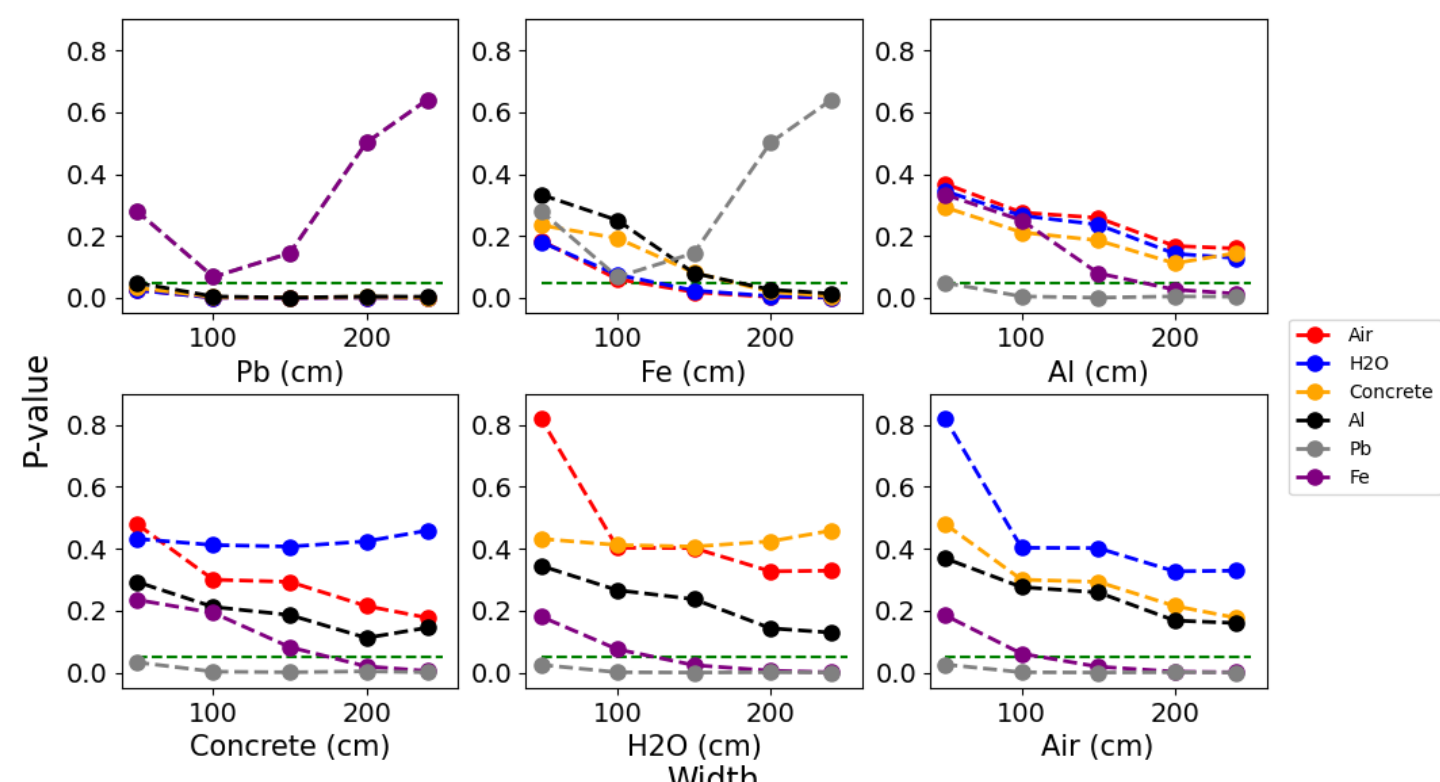

Figure 6: P-value calculated from the comparison between the distribution of scattering angles of different materials versus the width of these objects. The green line corresponds to $p$-value $=0.05$ as a limit to reject the null hypothesis. Each subplot corresponds to a different material with which the other materials are compared. 
The best geometrical parameters of the experimental setup that we found to differentiate materials using both tomography methods were $d=20 \mathrm{~cm}$ and $D=100 \mathrm{~cm}$ for the absorption method. In addition, using both methods we are able to distinguish a block of lead and iron from lighter materials as aluminum, concrete, water and air. Using the absorption method it is possible to identify lead at $5 \sigma$ when $W=150 \mathrm{~cm}$ and to differentiate iron when $W=250 \mathrm{~cm}$. However, in most cases, it is difficult to discern between lead and iron.

\section{Acknowledgements}

J. R. appreciates the support from the Peruvian National Council for Science, Technology and Technological Innovation scholarship under Grant 23-2015-FONDECyT. J. B. thanks the Dirección de Gestión de la Investigación (DGI - PUCP) for funding under Grant No. DGI-2019-3-0044.

\section{References}

[1] E.P. George and G.S. Shrikantia. Observations of the energy-spectrum of the cosmic radiation below ground. Nuclear Physics, 1:54-66, 1956.

[2] L. W. Alvarez and et. al. Search for hidden chambers in the pyramids. Science, 167(3919):832839, 1970.

[3] K. Morishima and et. al. Discovery of a big void in khufu's pyramid by observation of cosmic-ray muons. Nature, 552(7685):386-390, 2017.

[4] F. Ambrosino and et. al. The mu-ray project: detector technology and first data from $\mathrm{mt}$. vesuvius. Journal of Instrumentation, 9(02):C02029-C02029, 2014.

[5] W. J. Jo and et. al. Design of a muon tomography system with a plastic scintillator and wavelength-shifting fiber arrays. Nucl. Instrum. Meth. A, 732:2013, 568-572.

[6] S. N. Axani and et. al. The CosmicWatch Desktop Muon Detector: a self-contained, pocket sized particle detector. JINST, 13(03):P03019, 2018.

[7] S. N. Axani and et. al. The desktop muon detector: A simple, physics-motivated machineand electronics-shop project for university students. AJP, 85:948-958, 2017.

[8] L. Bonechi and et. al. Atmospheric muons as an imaging tool. Reviews in Physics, 5, 2020.

[9] G. R. Lynch and O. I. Dahl. Approximations to multiple coulomb scattering. Nucl. Instrum. Meth. B: Beam Interactions with Materials and Atoms, 58:6-10, 1991.

[10] S. Agostinelli and et. al. GEANT4-a simulation toolkit. Nucl. Instrum. Meth. A, 506:250-303, 2003. 